\title{
Relationship Between Bridging and Dimensions of Sella Turcica and Antero-Posterior Skeletal Malocclusions in Children
}

\author{
Seyed Hossein Moslemzadeh,, Negar Moghaddam,, Saeid Foroughi Moghaddam, ${ }^{1,}$ Ali Rafighi, \\ Morteza Ghojazadeh, ${ }^{3}$ and Farzaneh Rasouli ${ }^{1}$ \\ ${ }^{1}$ Department of Orthodontics, School of Dentistry, Tabriz University of Medical Sciences, Tabriz, IR Iran \\ ${ }^{2}$ Endodontic Department, School of Dentistry, Tabriz University of Medical Sciences, Tabriz, IR Iran \\ ${ }^{3}$ Assistant Professor of Physiology, Tabriz University of Medical Sciences, Tabriz, IR Iran \\ "Corresponding author: : Saeid Foroughi Moghaddam, Department of Orthodontics, School of Dentistry, Tabriz University of Medical Sciences, Tabriz, IR Iran. Tel: \\ +98-9125434124, E-mail: saidforoughi@gmail.com
}

Received 2016 February 19; Accepted 2016 April 25.

\begin{abstract}
Background: Interclinoid ligament calcification and dimensional changes in Sella Turcica seen on cephalometric radiographs are associated with some bone abnormalities as well as normal variations. Merging of anterior and posterior clinoid processes, known as Sella Turcica bridging or roofing and other changes in this area may occur as a part of large skeletal growth changes in body and may have association with craniofacial skeletal patterns development.

Objectives: The aim of the present study was to compare Sella Turcica bridging and dimensions of patients with various skeletal patterns to investigate whether there is a correlation between Sella Turcica region changes with skeletal patterns or not, and on the other hand, to know if these changes could be used as predictive indices for growing patients skeletal patterns.

Materials and Methods: A total of 108 cephalometric radiographs (36 Class I, 36 Class II, and 36 Class III) were randomly selected for evaluation. Sella Turcica was traced on acetate paper and scanned to produce digital images. The dimensions of Sella Turcica were measured with computer software using the Silverman, Kisling, and Axelsson method. To determine bridging, Leonardi's method was employed. To determine the association of Sella Turcica bridging and dimensions with different malocclusions, chi-squared test and one-way ANOVA were used.

Results: The means of Sella Turcica lengths in three groups were significantly different $(P=0.01)$. Mean depth and diameter, however, were not significantly different between malocclusions. In addition, bridging was not significantly different among three malocclusions evaluated.

Conclusions: According to the results, mean length of Sella Turcica, rather than depth and diameter, was significantly associated with the type of malocclusion. Sella Turcica cavity length is larger in $\mathrm{Cl}$ III patients in comparison with $\mathrm{Cl}$ I ones and may have predictive value in some instances.
\end{abstract}

Keywords: Sella Turcica, Malocclusion, Bridged, Radiography

\section{Background}

One of the most commonly used anatomic landmarks in the skull, which is used in cephalometric tracings, is the Sella point. This point is located at the center of Sella Turcica which encloses the pituitary gland at the base of the skull (1). Calcification of interclinoid ligaments (ICL) in the Sella Turcica, which are visible on cephalometric radiographs, is associated with some osseous abnormalities and normal variations $(2,3)$ and also some chronic systemic conditions are associated with morphological changes of Sella Turcica and for instance dysmorphologic types are more prevalent in diabetic patients (4). In addition, fusion of anterior and posterior clinoid processes, i.e. calcifica- tion of Sella Turcica might produce a closed and separate pituitary fossa view on cephalometric radiographs, which is called bridging or roofing of Sella Turcica $(3,5)$. The incidence rate of Sella Turcica bridging in normal individuals is $3.8-13 \%(3-6)$.

Some studies have shown changes in the shape of Sella Turcica during growth $(7,8)$. Anatomic abnormalities of Sella Turcica can be used as a diagnostic tool in interpretation of cephalometric radiographs (9). Since neural crest cells participate in the formation and development of Sella Turcica, teeth and the whole facial region, there might be a relationship between abnormalities in this region (3).

In 2000, Becktor evaluated bridging of Sella Turcica in patients with severe craniofacial disorders (7). In 2005, 
Jone evaluated bridging and dimensions of Sella Turcica in individuals who had been treated by orthodontic-surgical or orthodontic treatment modalities. The prevalence of Sella Turcica bridging in patients treated with orthodonticsurgical procedures was $16.7 \%$ while it was $7.3 \%$ in patients treated with only orthodontic methods (5). In 2007, Alkofide evaluated the morphology and size of Sella Turcica in class I, class II and class III patients in Saudi Arabia (1). In 2009, Marsan evaluated prevalence of Sella Turcica bringing and its dimensions in adult females in Turkey, who had skeletal class I and class III malocclusions. The results showed that class III individuals had a high prevalence rate of bridging; however, no differences were observed in Sella Turcica dimensions (10).

In 2010, Marcotty et al. evaluated Sella Turcica bridging in class I and class III patients. The results showed a higher prevalence of Sella Turcica bridging in class III patients (11).

Since most orthodontic patients undergo cephalometric radiographical examinations, morphologic changes in the Sella Turcica are easily diagnosed on these radiographs. If there is a relationship between morphologic changes of Sella Turcica and the type and severity of skeletal malocclusions, evaluation of these changes on cephalometric radiographs in growing children might help predict the quality and severity of malocclusion during adulthood and prepare a proper treatment plan.

\section{Objectives}

The aim of the present study was to evaluate and compare Sella Turcica bridging and dimensions in patients who had undergone cephalometric radiographic examinations for orthodontic treatment, with class I, class II and class III skeletal malocclusions.

\section{Materials and Methods}

In the present study the patient files in the archives of the department of orthodontics, Tabriz faculty of dentistry, were evaluated. The radiographs eligible to be evaluated in the study were selected and the subjects were divided into class I, class II and class III groups based on anteroposterior skeletal relationships. A total of 108 cephalometric radiographs ( 36 class I, 36 class II, and 36 class III) were randomly selected. Furthermore, each occlusal group was designed to have equal number of male and female participants. The criteria used to determine skeletal relationships were ANB angle and A-Pog distance according to values proposed by Anderson (12). ANB angle between the range $0.1^{\circ}-3.6^{\circ}$ who had also an A-Pog distance between 3.3-2.8 mm were considered as $\mathrm{Cl}$ I normal skeletal pattern, with greater amounts considered as $\mathrm{Cl}$ II and lesser ones as $\mathrm{Cl}$ III. Subsequently, 36 subjects were randomly selected in each group.

Inclusion criteria were the following:

1. 8 - 12 years of age

2. A high-quality lateral cephalometric radiograph

Exclusion criteria were the following items:

1. Cleft lip and/or cleft palate

2. Distinct craniofacial syndromes or anomalies

3. Severe skeletal disorders in the vertical dimension

4. Systemic hormonal imbalance or different amount of calcium intake or other systemic disease

Tracing of the Sella Turcica region was carried out on all the cephalometric radiographs, using acetate papers (Orthotechnology, USA) and 0.5-mm blue pencils. A Canon scanner (Model F 3200) was used to scan the tracings and save them in a computer in JPEG format and 1-to-1 magnification. In order to increase the accuracy of measurements, the computer images of tracings were evaluated using Corel DRAW (V.13) software to calculate Sella Turcica dimensions by Silverman technique at 0.01 accuracy (Figure 1).

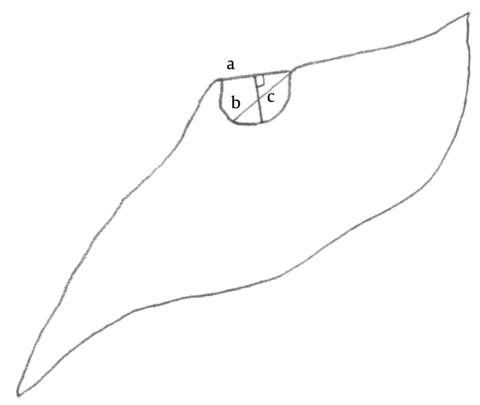

Figure 1. Calculation of Sella Turcica Dimensions Using Silverman Technique

In the technique introduced by Silverman the distance between the summit of Sella Turcica dorsum and the Sella Turcica tuberculum is measured (a), which shows the length of Sella Turcica. A vertical line (c) is drawn perpendicular to "a" from the deepest point on the sella turcica floor, which is considered the depth of sella turcica. The longest antero-posterior diameter of sella turcica is determined by measuring the distance between the sella turcica tuberculum and the most distant point on the inner surface of the posterior wall of sella turcica (b), which is referred to as the sella turcica diameter (Figure 2).

All the diagnostic reference points and dimensions were evaluated and controlled by an oral and maxillofacial radiologist. All the measurements were finally analyzed. Modified Leonardi technique was used to determine 

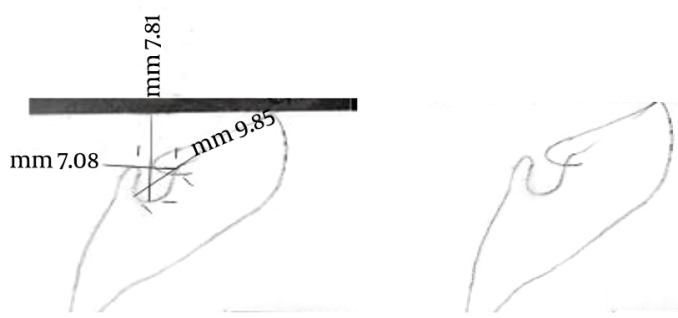

Figure 2. Measuring Sella Turcica Dimensions Using a Computer Software

bridging of Sella Turcica $(5,6)$. In this technique the length and diameter of Sella Turcica are measured. If the length is greater than three-quarters of the diameter, no calcification is suspected, referred to as Type I. If the length is less than the three-quarters of the diameter, there is partial calcification, referred to as Type II. If the diaphragm of Sella Turcica is visible on the radiograph or the distance between the anterior and posterior clinoid processes is equal to or less than $1 \mathrm{~mm}$, calcification is complete, referred to as Type III.

\subsection{Method Error}

In order to measure method error 10 samples were randomly selected from the three class I, class II and class III groups. After two weeks the tracing procedure was repeated by the same operator and also another operator, and measurements were repeated, too. MedCalC 15.2 software was used to calculate error between the two measurements for each sample in each group. This analysis revealed that the intraobserver and interobserver measurement repeatability was excellent (Intraclass correlation coefficient equal to 0.96).

\subsection{Statistical Analysis}

One-sample Kolmogorov-Smirnov test showed normal distribution of data. Sella Turcica dimension data were evaluated by descriptive statistical methods, including means \pm standard deviations. One-way ANOVA was used to compare Sella Turcica dimensions in skeletal malocclusions. Linear regression was used to evaluate relationship between age, gender and type of malocclusion on one hand and Sella Turcica dimensions on the other, using SPSS (V.13) statistical software. In case of statistical significance, a post hoc Tukey's test was used. Statistical significance was defined at $\mathrm{P}<0.05$.

Data about Sella Turcica bridging was analyzed using descriptive statistical methods (frequency, percent) and chi-squared test using SPSS (V.13) statistical software. Statistical significance was defined at $\mathrm{P}<0.05$.

\section{Results}

One-way ANOVA showed statistically significant differences in the means of Sella Turcica lengths between the three malocclusion groups $(\mathrm{P}=0.01, \mathrm{~F}(2,105)=4.83)$. The results of Tukey test revealed significant differences in the means of Sella Turcica lengths between class I and class III groups $(\mathrm{P}=0.01)$ with a greater length in $\mathrm{Cl}$ III. The means of Sella Turcica lengths between class I and class II groups $(\mathrm{P}=0.114)$ and between class II and class III groups $(\mathrm{P}=0.58)$ had no significant differences (Figure 3 ). However, there were no significant differences in the means of Sella Turcica diameters $(\mathrm{P}=0.61)$ and depths $(\mathrm{P}=0.37)$ between the three malocclusion groups.

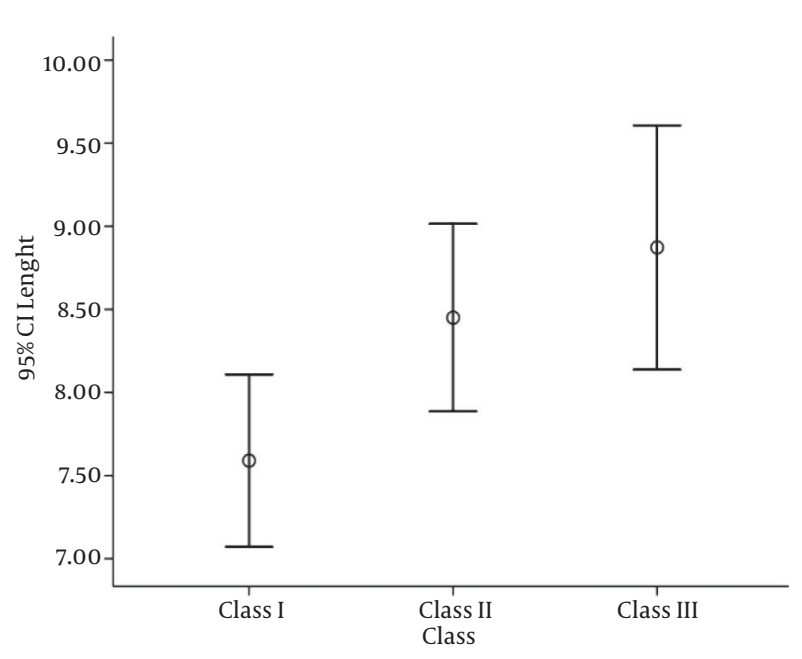

Figure 3. Means of Sella Tursica Length in Three Groups

The results of linear regression model showed that Sella Turcica length was significantly related to the skeletal relationship of the jaws $(\mathrm{P}=0.003)$. However, there was no relationship between gender and Sella Turcica dimensions (Tables 1 and 2).

In the present study the relationship between bridging and different types of malocclusion were evaluated in the antero-posterior dimension (Table 3). The results of chisquared test did not reveal any significant differences between the three skeletal relationships of the jaws $\left(\chi^{2}(4)=\right.$ $2.17, \mathrm{P}=0.70$ ).

\section{Discussion}

The present study evaluated and compared bridging and Sella Turcica dimensions in patients who had undergone cephalometric radiographic techniques for orthodontic treatment. The subjects were divided into class I 
Table 1. Descriptive Statistics Related to Sella Turcica Diameter $(P=0.61, F(2,105)=0.49)$

\begin{tabular}{|c|c|c|c|c|}
\hline Type of Malocclusion & Mean & SD & Min. & Max. \\
\hline Cl I & 10.85 & 1.39 & 10.37 & 11.32 \\
\hline Cl II & 11.14 & 1.56 & 10.61 & 11.66 \\
\hline Cl III & 10.86 & 1.20 & 10.46 & 11.27 \\
\hline
\end{tabular}

Table 2. Descriptive Statistics Related to Sella Turcica Depth $(P=0.37, F(2,105)=1.004)$

\begin{tabular}{|c|c|c|c|c|}
\hline Type of Malocclusion & Mean & SD & Min. & Max. \\
\hline $\mathrm{Cl} \mathbf{I}$ & 7.40 & 1.35 & 6.94 & 7.85 \\
\hline Cl II & 6.93 & 1.56 & 6.40 & 7.46 \\
\hline Cl III & 7.28 & 1.39 & 6.81 & 7.75 \\
\hline
\end{tabular}

Table 3. Relationship Between Bridging and Different Types of Malocclusion $\left(\chi^{2}(4)=2.17, \mathrm{P}=0.70\right)$.

\begin{tabular}{lccc}
\hline Type of Malocclusion & Type I bridging & Type II bridging & Type III bridging \\
\hline Cl I & $16(44.4 \%)$ & $17(47.2 \%)$ & $3(8.3 \%)$ \\
Cl II & $16(44.4 \%)$ & $16(44.4 \%)$ & $4(11.16 \%)$ \\
Cl III & $21(58.3 \%)$ & $13(36.1 \%)$ & $2(5.6 \%)$ \\
Total & $53(49.1 \%)$ & $46(42.6 \%)$ & $9(8.3 \%)$ \\
\hline
\end{tabular}

(normal skeletal pattern), class II, and class III skeletal malocclusion groups.

The results did not reveal any effect of gender on the length, depth and diameter of Sella Turcica. Since the subjects in the present study were very young ( 8 - 12 years of age) the relationship between age and Sella Turcica dimensions on one hand and bridging of Sella Turcica on the other was not evaluated. Similarly, Leonardi et al. had also concluded that interclinoid ligament calcification is not influenced by age and gender (3). The means of differences of the Sella Turcica lengths between class I and class III groups were statistically significant. The differences in Sella Turcica bridging were not significant between the three malocclusion groups.

Alkofide evaluated the shape and size of Sella Turcica in 11 - 16 year-old patients in Saudi Arabia. The results did not show any significant differences in the means of lengths, diameters and depths of Sella Turcica between males and females, which is consistent with the results of the present study. In the study carried out by Alkofide, the differences in the Sella Turcica diameters were significant between class II and class III malocclusions, with a greater diameter in class III malocclusion. In our study, mean differences of diameter and depth were not significantly different in the three groups, and there was only a significant statistical difference in Sella length between $\mathrm{Cl}$ I and $\mathrm{Cl}$ III groups, with a greater length in Cl III (1). So these two studies show Cl III malocclusion as having one aspect of Sella being greater to some extent. A recent review study by Sathyanarayana also reveals that the prevalence of Sella bridging is high in class III malocclusions and dental anomalies (13).

Becktor et al. evaluated Sella Turcica bridging in patients with severe craniofacial disorders and concluded that Sella Turcica bridging is highly prevalent in patients with severe craniofacial disorders (7). A total of 33 patients (18.6\%) of 177 subjects evaluated had Sella Turcica bridging. It should be emphasized that all the 177 subjects had severe craniofacial disorders. In the present study a total of 9 subjects (8.3\%) of 108 subjects evaluated had Sella Turcica bridging and none of them had severe craniofacial disorders. It should be pointed out that the technique used in the present study to diagnose Sella Turcica bridging was different from that used in the above-mentioned study.

Jone evaluated bridging and Sella Turcica dimensions in patients who had undergone orthodontic-surgical or simply orthodontic treatment modalities (5). The prevalence of bridging in patients receiving orthodonticsurgical treatment modalities was $16.7 \%$, with only $7.3 \%$ in patients receiving only orthodontic treatment modalities. The overall prevalence rate was not reported in that study.

Marsan evaluated prevalence of bridging and Sella Turcica dimensions in adult Turkish woman with class I 
and class III skeletal malocclusions. The results showed a higher prevalence of Sella Turcica bridging in individuals with class III malocclusion. This study, like above mentioned ones, also implies a relation between this type of malocclusion and one anatomic aspect of Sella. However, no significant differences were revealed in Sella Turcica dimensions between the two groups (10).

Marcotty et al. (11) evaluated Sella Turcica bridging in patients over 17 years of age, who had class I and class III malocclusions. The results showed a higher prevalence of bridging in class III patients.

The differences in the results of the present study and previous studies might be attributed to the fact that previous studies have evaluated adult patients but the subjects in the present study were 8 - 12 years of age (prepubescent subjects). In addition, it should be pointed out that in previous studies Becktor method has been used to diagnose Sella Turcica bridging; however, in the present study the method introduced by Leonardi was used.

It is suggested that the subjects in the present study be re-evaluated during adulthood and the results of the two studies be compared.

\subsection{Conclusion}

In prepubescent years there is a relationship between Sella Turcica length and different types of malocclusion shown by the difference in Sella length between $\mathrm{Cl} \mathrm{I}$ and $\mathrm{Cl}$ III groups, with a greater length in Cl III. However, no such relationship exists between different malocclusion types and diameter and depth of Sella Turcica. In addition, there is no relationship between Sella Turcica bridging and different types of malocclusion during these years.

\section{Footnotes}

Authors' Contribution: Seyed Hossein Moslemzadeh: supervisor; Negar Moghaddam: implementation; Saeid Foroughi Moghaddam: article writing and submitting; Ali Rafighi: supervisor; Morteza Ghojazadeh: statistical analysis; Farzaneh Rasouli: article revision.
Funding/Support: Tabriz University of Medical Sciences, Tabriz, IR Iran.

\section{References}

1. Alkofide EA. The shape and size of the sella turcica in skeletal Class I, Class II, and Class III Saudi subjects. Eur J Orthod. 2007;29(5):457-63. doi: 10.1093/ejo/cjm049. [PubMed: 17693429].

2. Kantor ML, Norton LA. Normal radiographic anatomy and common anomalies seen in cephalometric films. Am JOrthod Dentofacial Orthop. 1987;91(5):414-26. [PubMed: 3554978].

3. Leonardi R, Barbato E, Vichi M, Caltabiano M. A sella turcica bridge in subjects with dental anomalies. Eur J Orthod. 2006;28(6):580-5. doi: 10.1093/ejo/cjlo32. [PubMed:16954179].

4. Canigur Bavbek N, Dincer M. Dimensions and morphologic variations of sella turcica in type 1 diabetic patients. Am J Orthod Dentofacial Orthop. 2014;145(2):179-87. doi: 10.1016/j.ajodo.2013.10.011. [PubMed: 24485732].

5. Jones RM, Faqir A, Millett DT, Moos KF, McHugh S. Bridging and dimensions of sella turcica in subjects treated by surgical-orthodontic means or orthodontics only. Angle Orthod. 2005;75(5):714-8. doi: 10.1043/0003-3219(2005)75[714:BADOST]2.0.CO;2. [PubMed:16279819].

6. Axelsson S, Storhaug K, Kjaer I. Post-natal size and morphology of the sella turcica. Longitudinal cephalometric standards for Norwegians between 6 and 21 years of age. Eur J Orthod. 2004;26(6):597-604. doi: 10.1093/ejo/26.6.597. [PubMed: 15650069].

7. Becktor JP, Einersen S, Kjaer I. A sella turcica bridge in subjects with severe craniofacial deviations. Eur J Orthod. 2000;22(1):69-74. [PubMed: 10721247]

8. Bjork A. Cranial base development. Am J Orthod. 1955;41(3):198-225. doi: 10.1016/0002-9416(55)90005-1.

9. Cederberg RA, Benson BW, Nunn M, English JD. Calcification of the interclinoid and petroclinoid ligaments of sella turcica: a radiographic study of the prevalence. Orthod Craniofac Res. 2003;6(4):22732. [PubMed: 14606526].

10. Marsan G, Oztas E. Incidence of bridging and dimensions of sella turcica in Class I and III Turkish adult female patients. World J Orthod. 2009;10(2):99-103. [PubMed: 19582250].

11. Meyer-Marcotty P, Reuther T, Stellzig-Eisenhauer A. Bridging of the sella turcica in skeletal Class III subjects. Eur J Orthod. 2010;32(2):14853. doi: 10.1093/ejo/cjp081. [PubMed: 19752019].

12. Anderson G, Fields HW, Beck M, Chacon G, Vig KW. Development of cephalometric norms using a unified facial and dental approach. Angle Orthod. 2006;76(4):612-8. doi: 10.1043/00033219(2006)076[0612:DOCNUA]2.0.CO;2. [PubMed: 16808567].

13. Sathyanarayana HP, Kailasam V, Chitharanjan AB. Sella turcica-Its importance in orthodontics and craniofacial morphology. Dent Res J (Isfahan). 2013;10(5):571-5. [PubMed: 24348611]. 